\title{
Nonlinear Analysis of Electrically Actuated Carbon Nanotube Resonator Using a Novel Discretization Technique
}

\author{
Hassen M. Ouakad, ${ }^{1}$ Fehmi Najar, ${ }^{2}$ and Oussama Hattab ${ }^{2}$ \\ ${ }^{1}$ Mechanical Engineering Department, King Fahd University of Petroleum and Minerals, P.O. Box 31261, Dhahran, Saudi Arabia \\ ${ }^{2}$ Applied Mechanics and Systems Research Laboratory, Tunisia Polytechnic School, University of Carthage, BP 743, \\ 2078 La Marsa, Tunisia
}

Correspondence should be addressed to Hassen M. Ouakad; houakad@kfupm.edu.sa

Received 21 April 2013; Revised 22 August 2013; Accepted 22 August 2013

Academic Editor: Sarp Adali

Copyright (C) 2013 Hassen M. Ouakad et al. This is an open access article distributed under the Creative Commons Attribution License, which permits unrestricted use, distribution, and reproduction in any medium, provided the original work is properly cited.

Static as well as dynamic analyses have been performed on clamped-clamped carbon nanotube (CNT) resonator. The nonlinear CNT model is investigated with a novel discretization technique: a differential quadrature method (DQM) to discretize the spatial variables and a finite difference method (FDM) for limit-cycle solutions. Parametric study is performed by varying the electric load, as well as the initial curvature (due to fabrication). It is found that the pull-in voltage decreases nonlinearly with initial curvature and linearly with residual stresses. The eigenvalue problem is also solved to obtain the bending natural frequencies of the system as function of the DC voltage as well as the initial curvature of the CNT. Frequency-response curves near some selected resonant frequencies are plotted to better understand the nanotubes' dynamic behavior. Different linear and nonlinear phenomena are depicted such as dynamic pull-in, hardening, and softening behavior and veering of the odd modes. We have found that even when exciting the CNT near its first natural frequency, the vibration mode located at the veering process significantly alters the CNT's motion and hence may decrease its overall quality factor.

\section{Introduction}

Since their discovery [1], carbon nanotubes (CNTs) are in the center of interest of researchers thanks to their revolutionary characteristics such as high Young's modulus and low density leading to light structures with high mechanical performance. In addition to that, promising applications in several fields like optics, electronics, and resonant sensors are investigated. Their natural frequencies are found to be very high compared to other structures, which permit to design high frequency resonators [2].

Static and dynamic analyses of CNTs rise difficult issues in solving the highly nonlinear governing equation. In this case the nonlinearity is both of the geometric type (midplane stretching) and of the loading type (electric load) [3]. Several methods were attempted to solve the problem using the Galerkin procedure along with shooting techniques [4] or molecular dynamics simulations $[5,6]$.
Previous studies have focused on mechanical as well as electric response of CNTs exploring their interesting characteristics such as Young's modulus and pull-in properties. Experimental investigations of the vibration of electrostatically actuated CNTs have been conducted to characterize their effective Young modulus (Poncharal et al. [7]). On the other hand, others have found that Van der Waals effect on static response of CNTs is negligible for gap widths greater than $3 \mathrm{~nm}[5,6]$. Using the reduced order model (ROM) with the Galerkin method, Ouakad and Younis [4] have investigated the effect of geometric parameters and initial curvature on pull-in and the dynamic response of CNTs. They are found to hold a hardening behavior in almost all of the cases until applied voltage is high enough (near pullin) to change the dynamic response into softening type. On the other hand, cantilever beams are found to hold softening behavior [4]. Natural frequencies variation with initial curvature has revealed the existence of phenomenon 
called modes veering. Ouakad and Younis [4] investigated the effect of van der Waals forces on the fundamental natural frequency of CNTs as well and found them significant for CNTs of very small gap widths.

Using the nonlocal Timoshenko model, the effect of temperature change on mode shapes is investigated [8]. Studying the dynamic response of an array of nanotubes has revealed that interaction between tubes effects considerably the mode shapes and their stability [9]. Nonlinear bifurcations have been shown to result in a transition from quasi-periodic to chaotic behavior in dynamic response [10]. Double-walled carbon nanotubes (DWCNTs) have been analyzed by Fang et al. [11] determining the effect of different nonlinearities on their dynamic response and by Hajnayeb and Khaddem [12] who found that both layers vibrate with the same frequency. Huang [13] has investigated the effects of adhesion temperature, tube length, and peeling velocity on the dynamic behavior of a DWCNT on an Au substrate.

Many researchers have fabricated and tested systems based on CNTs such as torsional electromechanical systems [14], strain sensors [15], biosensors [16, 17], and pH sensing system [18]. For example, Cho et al. [19] have designed a cantilever nanotube which has been tested successfully using multiscale analysis with comparison to experimental results. The cantilever nanotube is found to be sensitive to geometric parameters changes altering the dynamic response from hardening behavior to softening behavior. Graham et al. [20] have applied the 2D Fourier transform spectroscopy to semiconducting SWCNTs determining spectral and timedomain map of exciton-phonon assisted excitations. Several studies have focused on the energetic behavior of CNTs. As examples, Peng et al. [21] have studied experimentally using TEM the dissipation of energy due to air damping and contact loss. Greaney and Grossman [22] have used molecular dynamics to simulate energy transfer between nanotubes and have found that efficient energy transfer is allowed by sharp resonance effects.

The objective of the paper is to solve both static and dynamic nonlinear problems using novel discretization technique: differential quadrature method and finite difference method. These methods have been applied successfully to MEMS electrostatic actuators in order to investigate their nonlinear dynamics [23-25]. The considered model accounts for geometrical through the midplane stretching and also takes into account the fully nonlinear form of the electrostatic force. In this paper, we present the proposed ROM by applying the DQM. Then, free-vibration problem under the actuation of DC load is solved in order to predict natural frequencies and mode shapes of the system. Finally, we attempt to give a response to dynamic transformations occurring during veering phenomenon by plotting the frequencyresponse curves under $\mathrm{AC}$ and DC loading around different excitation frequencies.

\section{Problem Formulation and Reduced-Order Model}

2.1. Problem Formulation. A hollow cylindrical EulerBernoulli beam of length $l=3000 \mathrm{~nm}$, shell thickness $h=0.34 \mathrm{~nm}$, radius $\widehat{R}=1 \mathrm{~nm}, d=500 \mathrm{~nm}$ is considered in this paper to model an electrostatic CNT resonator initially curved in the direction of the lower electrode [26] (Figure 1). It is assumed to have Young's modulus $E=1.2 \mathrm{TPa}$ [7] and a mass density $\rho=1.3 \mathrm{~g} / \mathrm{cm}^{3}$ [27]. The mathematical formulation of the static and dynamic behaviors of the clamped-clamped CNT in its nondimensional form is given by $[4]$

$$
\begin{aligned}
\frac{\partial^{4} w}{\partial x^{4}} & +\frac{\partial^{2} w}{\partial t^{2}}+c \frac{\partial w}{\partial t} \\
= & \alpha_{2} F_{e}+\left[N-\alpha_{1} \int_{0}^{1}\left(w \frac{\partial^{2} w}{\partial x^{2}}-2 \frac{\partial w}{\partial x} \frac{\partial w_{0}}{\partial x}\right) d x\right] \quad \\
& \times\left(\frac{\partial^{2} w}{\partial x^{2}}+\frac{\partial^{2} w_{0}}{\partial x^{2}}\right), \\
w(0, t) & =0, \quad w(1, t)=0, \quad \frac{\partial w}{\partial x}(0, t)=0, \quad \frac{\partial w}{\partial x}(1, t)=0
\end{aligned}
$$

where the longitudinal coordinates $x$ and time $t$ are normalized with respect to the length $l$ and the time constant $T$, respectively. $w(x, t)$ is the nondimensional transverse deflection toward the substrate, the initial shape function is $w_{0}(x)=\left(b_{0} / d\right) \sin (\pi x)[28,29]$ and

$$
\begin{aligned}
& F_{e}=\left(\left(V_{\mathrm{DC}}+V_{\mathrm{AC}} \cos (\Omega t)\right)^{2}\right) \\
& \times\left(\sqrt{\left(1-w-w_{0}\right)\left(1-w-w_{0}+2 R\right)}\right. \\
&\left.\times\left(\cosh ^{-1}\left(1+\frac{1-w-w_{0}}{R}\right)\right)^{2}\right)^{-1}, \\
& N=\frac{\widetilde{N} l^{2}}{E I}, \quad \alpha_{1}=\frac{A d^{2}}{2 I}, \quad \alpha_{2}=\frac{\pi \epsilon_{0} l^{4}}{E I d^{2}}, \\
& c=\frac{\rho A \omega}{Q} \frac{l^{4}}{E I}, \quad T=\sqrt{\frac{\rho A l^{4}}{E I}}, \quad R=\frac{\widehat{R}}{d},
\end{aligned}
$$

where $A=\pi(R+(h / 2))^{2}-\pi(R-(h / 2))^{2}$ is the cross-section area, $I=(\pi / 4)\left((R+(h / 2))^{4}-(R-(h / 2))^{4}\right)$ is the moment of inertia, $\omega$ is the natural frequency, $\widetilde{N}$ is the axial force modeling the residual stress, and $Q$ is the quality factor.

\subsection{Reduced-Order Model Using the Differential Quadrature} Method. The differential quadrature method (DQM) is used to solve the space dependent partial differential equation by transforming it into ordinary differential equations describing the motion of the CNT with respect to time at $n$ preselected grid points $x_{i}=(1 / 2)[1-\cos (((i-1) /(n-1)) \pi)]$. The derivatives of the deflection with respect to space variable are expressed as a weighted linear sum of the deflection at all grid points. Following [23], we discretize the integral terms using the Newton-Cotes formula at the same grid points. 


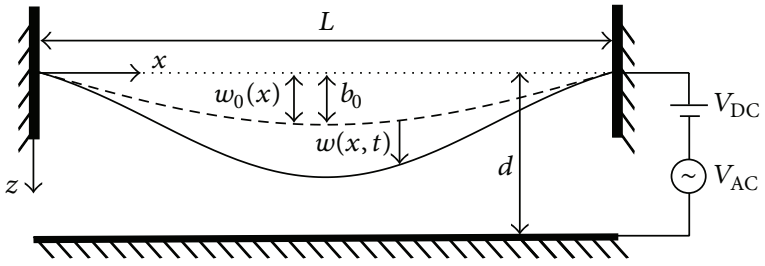

FIGURE 1: Schematic of the CNT resonator.

Using the boundary conditions given in (2), we end up with the following $(n-4)$ ODEs,

$$
\begin{aligned}
\ddot{w}_{i}+ & c \dot{w}_{i}+\sum_{j=1}^{n} A_{i j}^{(4)} w_{j} \\
= & {\left[N-\alpha_{1} \sum_{j=1}^{n} \sum_{k=1}^{n} C_{j} A_{j k}^{(2)} w_{k}\left(w_{j}-2 w_{0}^{\prime}\left(x_{j}\right)\right)\right] } \\
& \times\left[\sum_{j=1}^{n} A_{i j}^{(2)} w_{j}+w_{0}^{\prime \prime}\left(x_{i}\right)\right] \\
& +\frac{\alpha_{2}}{\sqrt{\left(1-w_{i}-w_{0}\left(x_{i}\right)\right)\left(1-w_{i}-w_{0}\left(x_{i}\right)+2 R\right)}} \\
& \times\left(\frac{V_{\mathrm{DC}}+V_{\mathrm{AC}} \cos (\Omega t)}{\operatorname{arccosh}\left(1+(1 / R)\left(1-w_{i}-w_{0}\left(x_{i}\right)\right)\right)}\right)^{2},
\end{aligned}
$$

$w_{i}=w\left(x_{i}, t\right)$ and the coefficients $C_{i}$ and $A_{i j}^{(r)}$ are given in [23].

\section{Static Analysis}

3.1. Convergence and Validation. In this section, we solve the algebraic system obtained by dropping the time dependent terms in (4). First, we perform a convergence study in which the static response of the CNT is calculated by varying the number of grid points $n$ varied from 5 to 15 . The results are compared to those obtained by Ouakad and Younis [29] and calculated using the Galerkin method. In Figure 2, we show that the number of grid points required to have converged results is 11. Hence, in all of the following results, we will be using at least 11 points in the DQM to assure convergence.

3.2. Effect of Residual Stress. Residual stresses have thermal origins due to fabrication techniques of the CNT [30]. We model this effect here by adding an axial force constant along the length of the CNT. We study here the influence of these residual stresses on the static response of the CNT for both compression $(N \leq 0)$ and tension $(N \geq 0)$ cases. It is always assumed to be lower than the first buckling load $\widetilde{N}_{b}=$ $\left(4 \pi^{2} E I\right) / l^{2}$.

In Figure 3 we show that residual stresses have a relatively small effect on the static response of the CNT. Indeed an increase of the residual stress induces an increase of the pullin voltage for different values of the initial curvature.

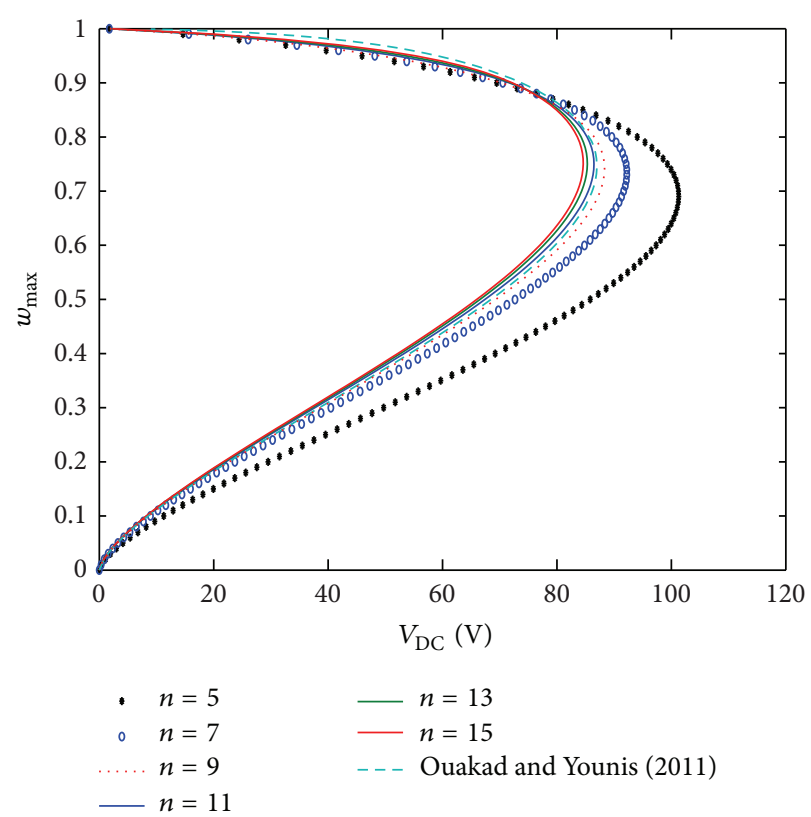

FIGURE 2: Variation of the normalized static deflection with DC voltage for different numbers of grid points.

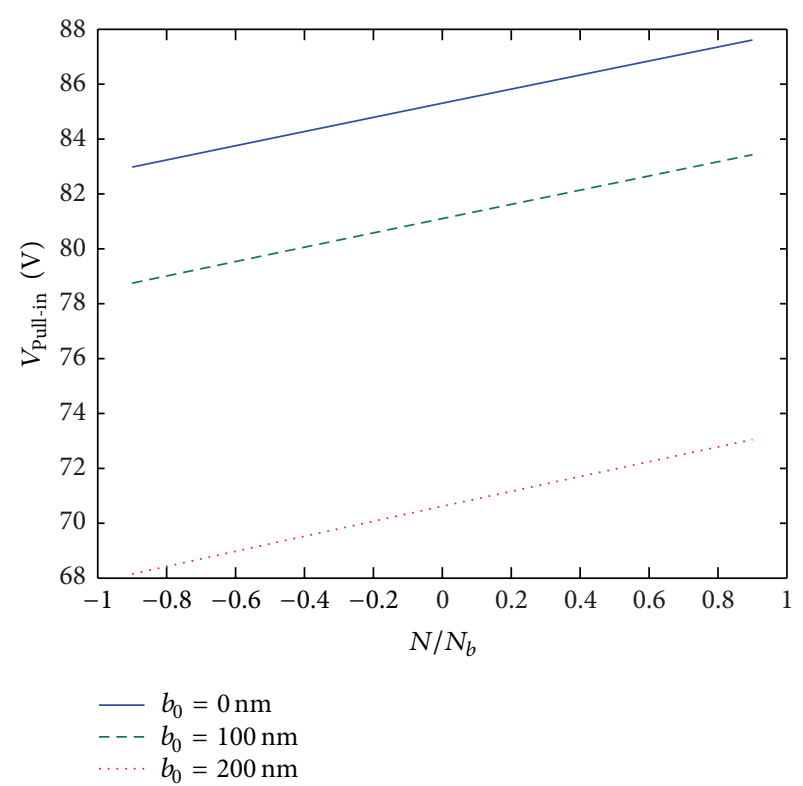

FIGURE 3: Variation of the pull-in voltage with the residual stresses at different initial curvatures.

3.3. Effect of Initial Curvature. We investigate next the effect of the initial curvature into the static response of the CNT. The initial curvature $b_{0}$ is supposed to vary from $100 \mathrm{~nm}$ to $200 \mathrm{~nm}$.

The results in Figure 4 are in a good agreement with results reported in [29]. In Figure 5, we show that pull-in voltage is lowered when initial curvature is higher. This is due to the fact that in the initially curved configuration, the beam is closer to the electrode which means that, at the same level of voltage, electrostatic force is higher compared to elastic force. 


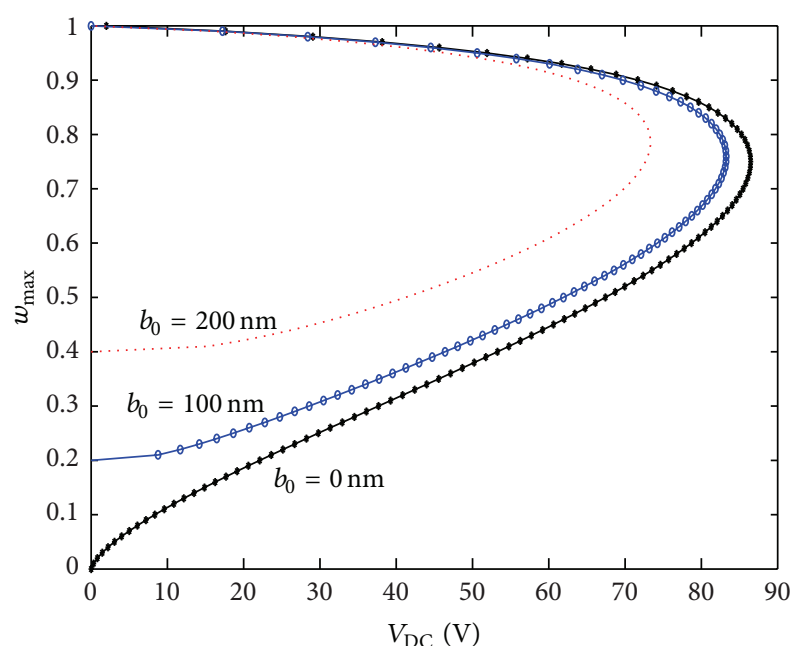

FIGURE 4: Variation of the normalized static deflection with DC voltage for different initial curvatures $b_{0}$.

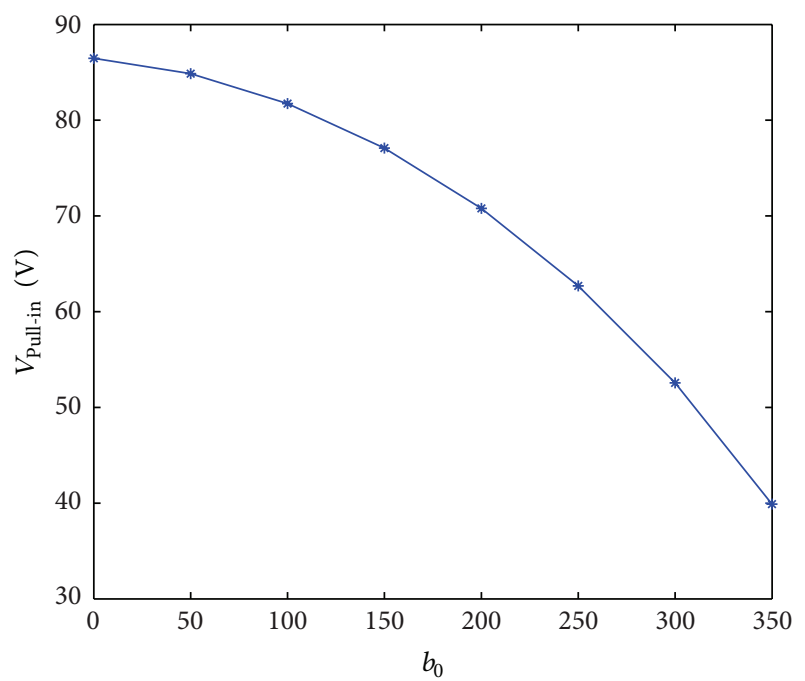

FIGURE 5: Variation of the pull-in voltage with the initial curvatures $b_{0}$.

\section{Natural Frequencies and Mode Shapes of the Curved CNT under DC Voltage}

Assuming that superposition principle applies to the CNT deflection, we split it into static part due to DC voltage and dynamic part due to AC voltage assumed to be harmonic, that is, $w(x, t)=w^{s}(x)+u(x, t)=w^{s}(x)+\phi(x) e^{\imath \omega t}$. For the DQM form, we replace the latter expression by its value at node $i$; that is, $w_{i}(t)=w_{i}^{s}+u_{i}(t)=w_{i}^{s}+\phi_{i} e^{i \omega t}$. Then we use (4) and drop the damping and forcing AC terms. The electrostatic force is linearized using the Taylor series to the first order around its static solution [25]. Finally, we obtain the following eigenvalue problem of order $(n-4)$ :

$$
\begin{aligned}
& \sum_{j=1}^{n} A_{i, j}^{(4)} \phi_{j}-\omega^{2} \phi_{i}-\left.\phi_{i} \frac{\partial F_{e}}{\partial w}\right|_{w=w_{i}^{s}}-N \sum_{j=1}^{n} A_{i, j}^{(2)} \phi_{j} \\
& +2 \alpha_{1} \sum_{j=1}^{n} \sum_{p=1}^{n} \sum_{q=1}^{n} C_{p} A_{p, q}^{(1)} A_{i, j}^{(2)} w_{j}^{s} \\
& \quad \times\left(w_{0}^{\prime}\left(x_{p}\right) \phi_{q}-\sum_{r=1}^{n} w_{q}^{s} A_{p, r}^{(1)} \phi_{r}\right) \\
& +\alpha_{1} \sum_{j=1}^{n} \sum_{p=1}^{n} \sum_{q=1}^{n} w_{q}^{s} A_{i, j}^{(2)} \phi_{j} C_{p} \\
& +2 \alpha_{1} w_{0}^{\prime \prime}\left(x_{i}\right) \\
& \times \sum_{p=1}^{n} \sum_{q=1}^{n} C_{p} A_{p, q}^{(1)}\left(2 w_{0}^{\prime}\left(x_{p}\right) A_{p, q}^{(1)}+w_{p}^{s} A_{p, q}^{(2)}\right) \\
& \times\left(w_{0}^{\prime}\left(x_{p}\right) \phi_{q}-\sum_{r=1}^{n} w_{q}^{s} A_{p, r}^{(1)} \phi_{r}\right)=0 \\
& i=3, \ldots, n-2,
\end{aligned}
$$

which can be reduced to the following matrix form:

$$
\mathbf{A} \Phi=\omega^{2} \Phi
$$

where $\Phi=\left(\phi_{3} \cdots \phi_{n-2}\right)^{T}$. The natural frequencies are the square roots of the eigenvalues of the matrix $\mathbf{A}$, and the mode shapes are the corresponding eigenvectors.

4.1. Effect of Initial Curvature on the Natural Frequencies. At a given applied DC voltage, we investigate the effect of variation of initial curvature on natural frequencies described by the parameter $b_{0}$. The results are summarized in Figure 6 .

We can see clearly that even frequencies are insensitive to the variation of curvature, whereas the odd frequencies vary with slack. One can see that odd frequencies do not intersect, but they diverge in a manner called frequency or mode veering [29]. A frequency veering occurs when the loci of two frequencies approach each other when a parameter is varied and then veer away when being too close (similar to the repulsive charges process). Adding to that, we can also clearly see that the odd frequencies intersect the even ones for certain levels of slack offering many possibilities of internal resonances and exchange of energy among higher- and lowerorder modes [31].

The same phenomenon of mode veering is observed in Figure 7. This behavior will be further investigated in the next section by exploring the dynamic behavior of the CNT before and after veering.

4.2. Effect of DC Voltage on the Natural Frequencies. For initial curvature $b_{0}=100 \mathrm{~nm}$, we vary the applied DC 


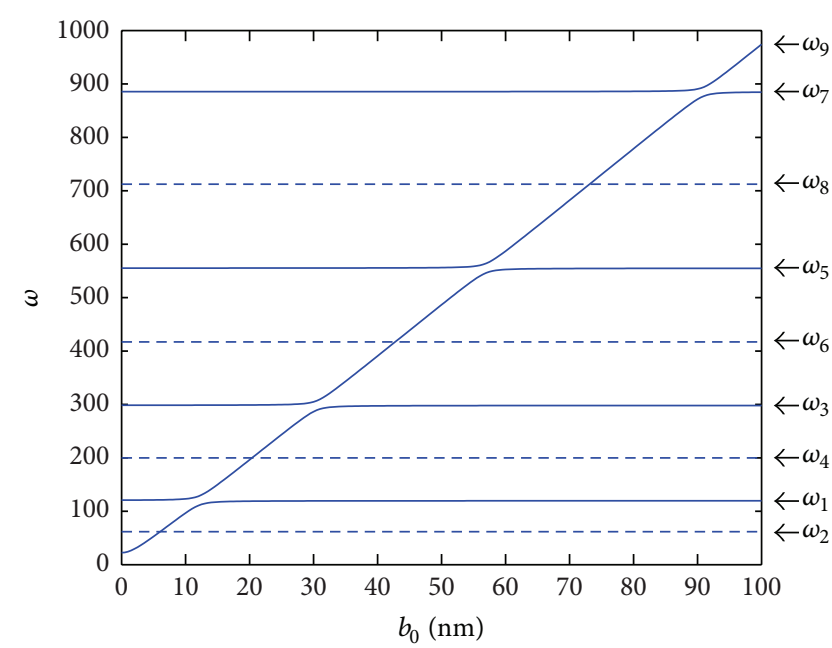

FIGURE 6: Variation of the 9 first natural frequencies with $b_{0}$ at $V_{\mathrm{DC}}=0 \mathrm{~V}$.

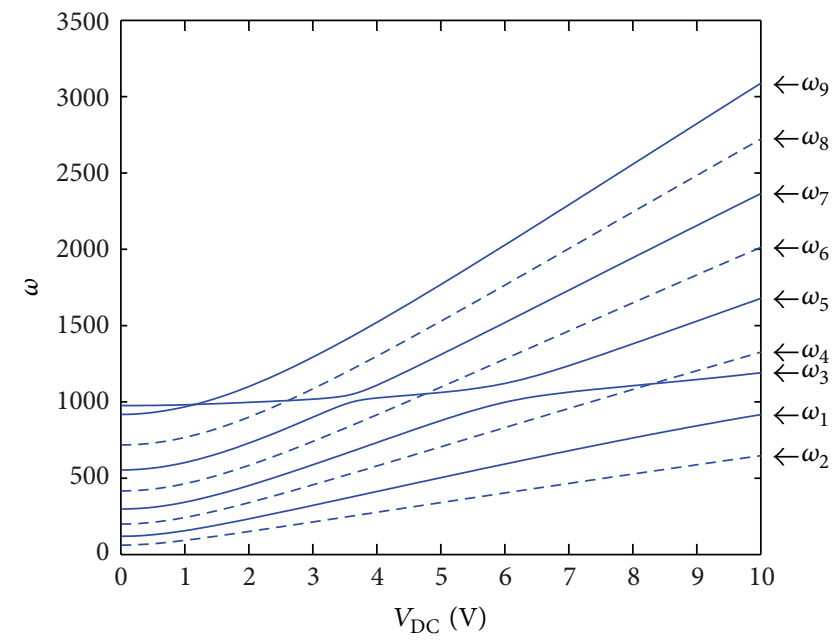

FIGURE 7: Variation of the 9 first natural frequencies with DC voltage at $b_{0}=100 \mathrm{~nm}$.

voltage and investigate its effect on the natural frequencies. The results are summarized in Figure 7. It shows that natural frequencies of the CNT increase as function of DC voltage exhibiting a hardening-type behavior. This behavior perseveres until pull-in where the natural frequencies drop down. Also from the same figure, two consecutive odd natural frequencies approach each other as the DC voltage is varied and then diverge; this is known as mode veering.

4.3. CNT Mode Shapes Interchangeability. To further clarify the veering process with the DC voltage depicted in Figure 7, we plot the eigenfunctions (mode shapes) of the CNT natural frequencies for $V_{\mathrm{DC}}=5 \mathrm{~V}$, Figure 8 . In a frequency veering process, the eigenfunctions associated with the eigenvalues on each locus before veering are interchanged during the veering. For example, in Figure 8, we can clearly see that mode number 5 is the one that has the shape of the fundamental clamped-clamped beam mode along with its fifth mode.
Now, and as seen before in Figure 6, the shape of the first mode is transferred into the different odd modes, from the higher to the lower modes as the DC voltage is increased till it is transmitted to the lowest fundamental frequency, which eventually drops to zero at pull-in, a thing very similar to what was reported in [29] when varying the CNT slack level.

\section{Dynamic Response and Limit-Cycle Solution of the CNT}

In this section, we present the dynamic behavior of the CNT by generating their frequency-response curves for different sets of parameters. For this, we have to solve the dynamic equation (4) obtained by the DQM. For $n$ grid points and using the boundary conditions and the symmetry of the problem, we end up with $(n-$ 3 )/2 ODEs describing the motion of the system. To seek limit-cycle solutions, we assume that the periodic orbits have the same period than the excitation $(\Omega)$. The time is discretized along the assumed period, and the ODEs obtained by the DQM are discretized in time using the FDM. In addition, we enforce the condition that the first and last solutions along the orbit are equal; this will satisfy the periodicity condition of the limit-cycle solution.

To construct the limit-cycle solutions and the frequencyresponse curves of the CNT, we seek periodic orbits of the system with period $T=2 \pi / \Omega$. In implementing the FDM, we rescale the time so that the period is unity and discretize the orbit by $(m+1)$ points; thus, $\Delta t=1 / m$. To guarantee periodicity of the orbit, we impose the condition that the first and last points of the orbit (points 1 and $m$ ) are identical. We end up with the following system of $(\mathrm{N}-$ $1)(n-3)$ nonlinear algebraic equation, which is solved using the Newton-Raphson method [25]:

$$
\begin{aligned}
& \dot{w}_{i, p}=w_{i, p}^{v}, \\
& \dot{w}_{i, p}^{v}=- {\left[c w_{i, p}^{v}+\sum_{j=1}^{n} A_{i j}^{(4)} w_{j}\right.} \\
&-\frac{\alpha_{2}}{\sqrt{\left(1-w_{i}-w_{0}\left(x_{i}\right)\right)\left(1-w_{i}-w_{0}\left(x_{i}\right)+2 R\right)}} \\
&\left.-\left[N-\alpha_{1} \sum_{j=1}^{n} \sum_{k=1}^{n} C_{j} A_{j k}^{(2)} w_{k}\left(w_{j}-2 w_{0}^{\prime}\left(x_{j}\right)\right)\right]\right], \\
& \quad i=3,4, \ldots, \frac{1}{2}(n+1), \quad p=1,2, \ldots, m,
\end{aligned}
$$

where $t_{p}=p \Delta t, w_{i, p}=w\left(x_{i}, t_{p}\right)$, and $w_{i, p}^{v}=w^{v}\left(x_{i}, t_{p}\right)=$ $\dot{w}\left(x_{i}, t_{p}\right)$.

5.1. Frequency-Response Curves of the CNT. The frequencyresponse curves are obtained by plotting the variation of the 

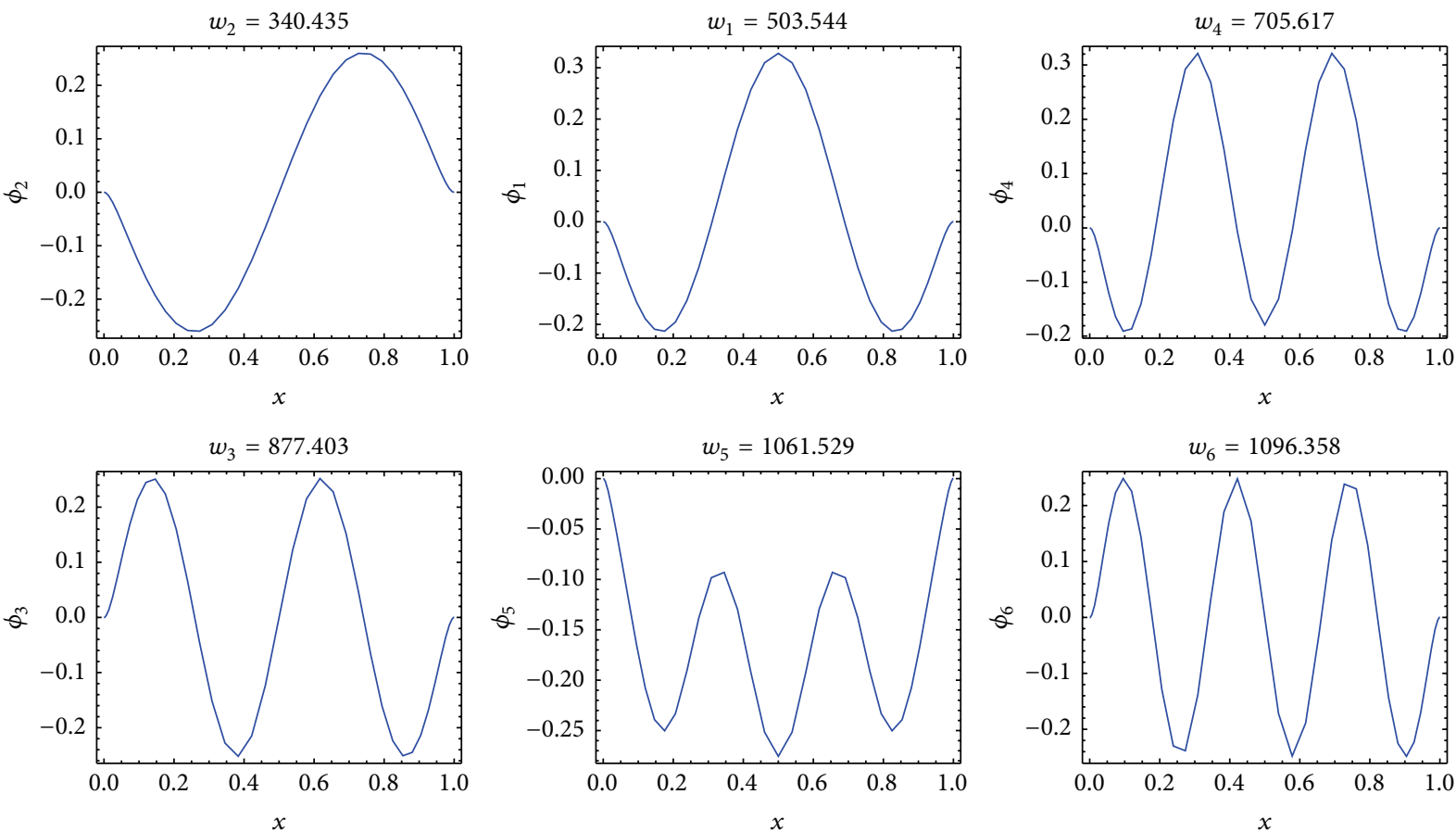

FIGURE 8: Mode shaped of the CNT without the initial curvature for $b_{0}=100 \mathrm{~nm}, V_{\mathrm{DC}}=5 \mathrm{~V}$, and 41 grid points.
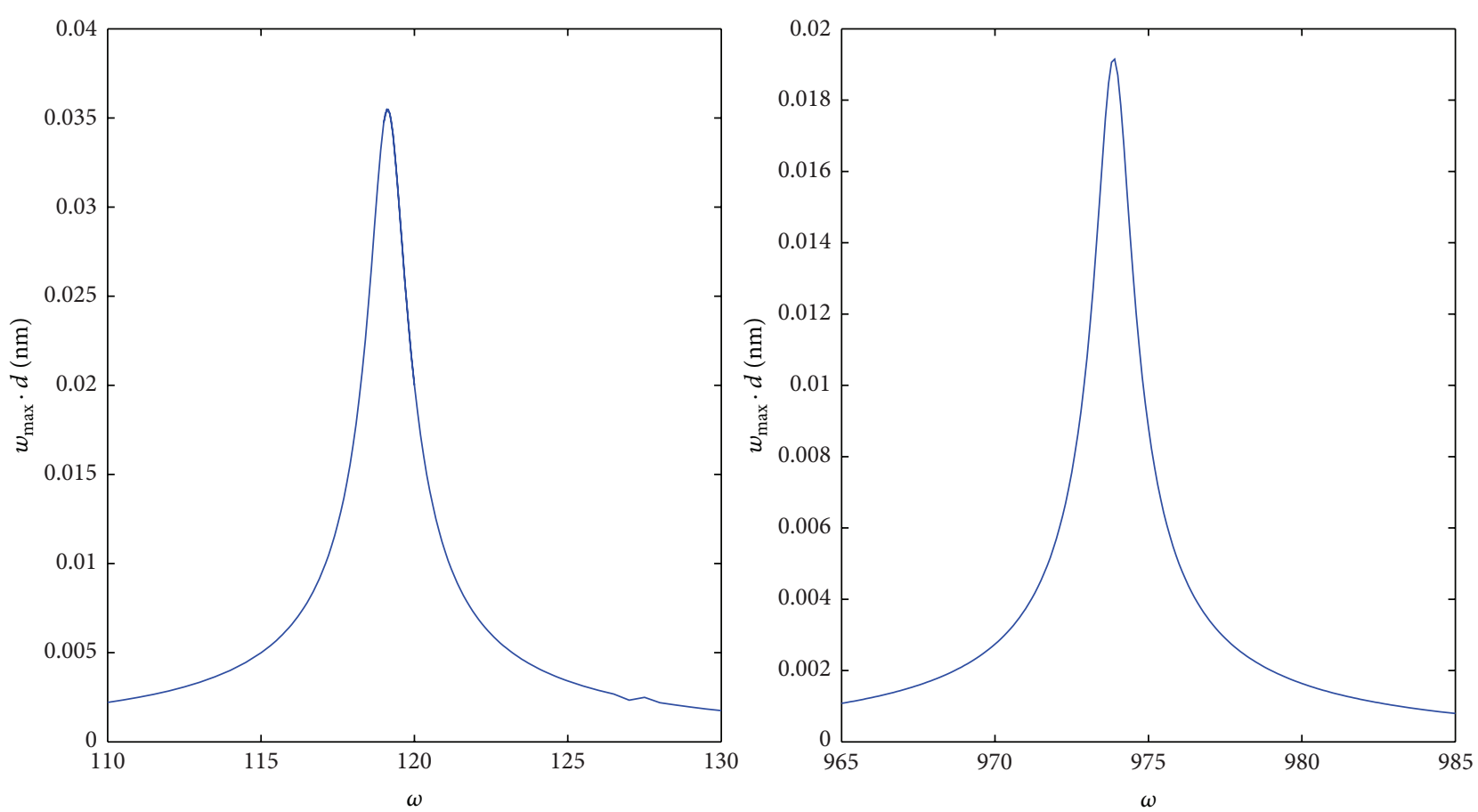

FIGURE 9: Frequency-response curves near the 1st and 9th natural frequencies when $b_{0}=100 \mathrm{~nm}$. Results are shown for $V_{\mathrm{DC}}=0.01 \mathrm{~V}$, $V_{\mathrm{AC}}=0.01 \mathrm{~V}$, and $\mathrm{Q}=100$. 

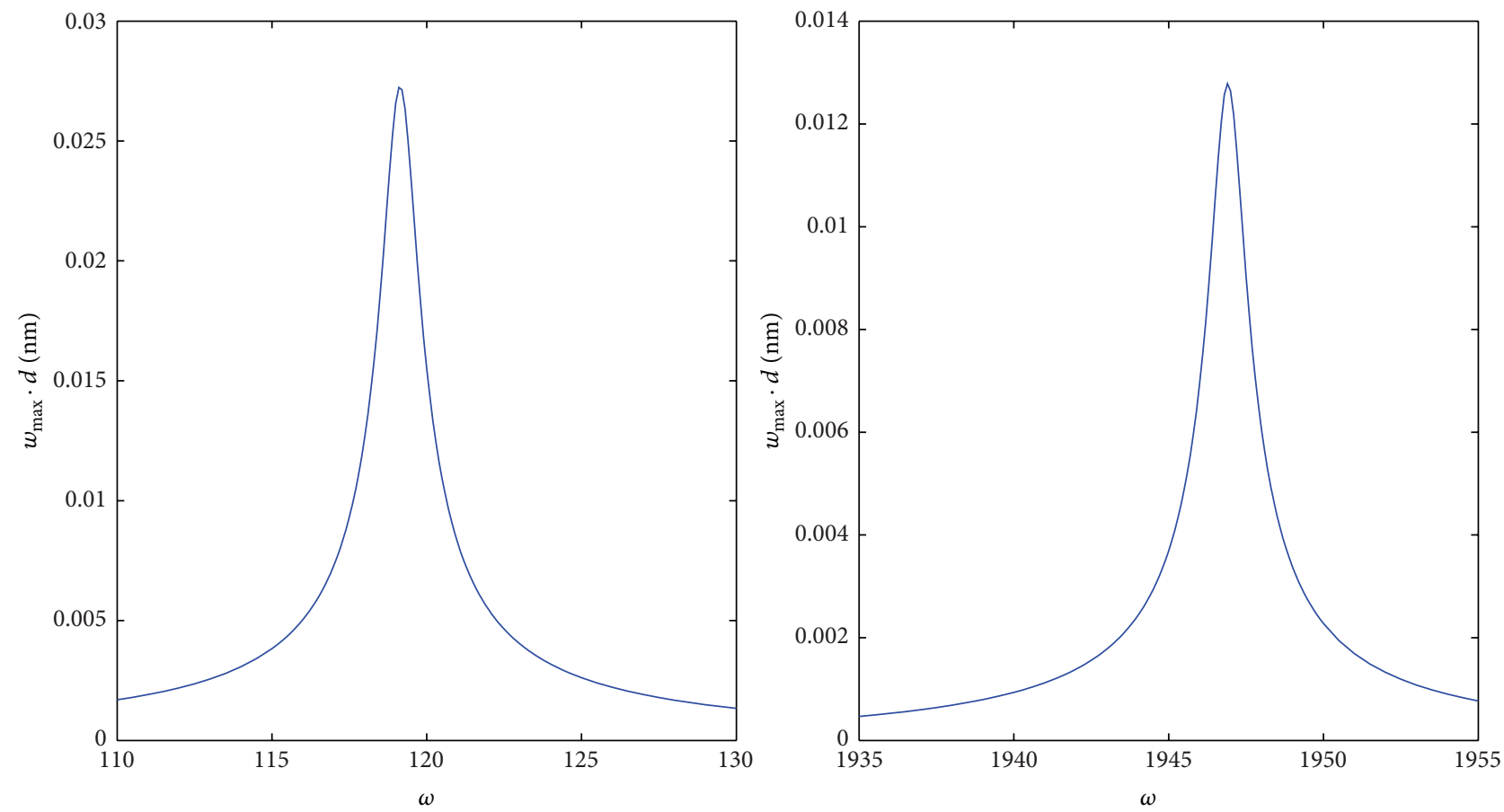

FIGURE 10: Frequency-response curves of $200 \mathrm{~nm}$ slack showing peaks at the 1st and 11th natural frequencies. Results are shown for $V_{\mathrm{DC}}=$ $0.01 \mathrm{~V}, V_{\mathrm{AC}}=0.01 \mathrm{~V}$, and $Q=100$.

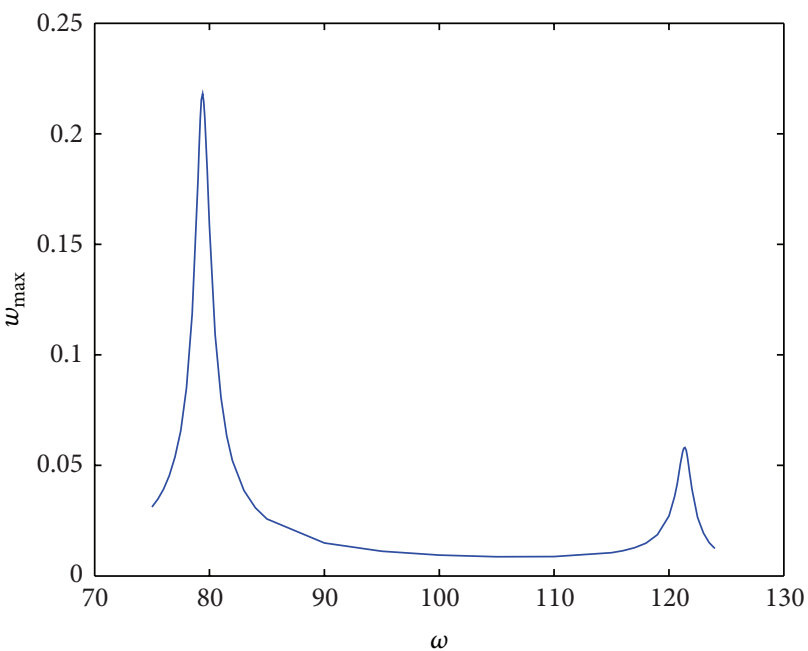

(a)

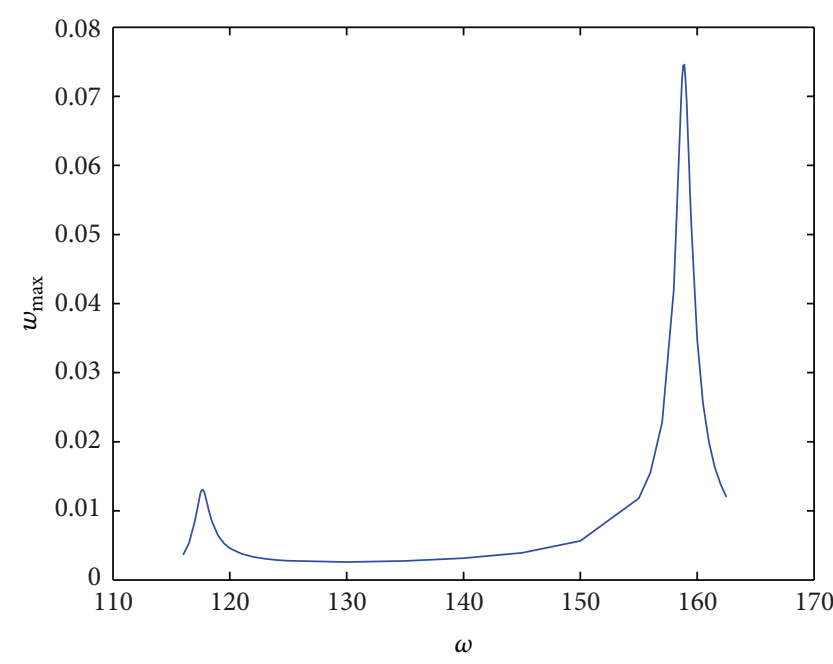

(b)

FIGURE 11: Frequency response near the first and third natural frequencies when first mode is excited before veering at (a) $b_{0}=8 \mathrm{~nm}$ and after veering (b) $b_{0}=16 \mathrm{~nm}$.

maximum deflection of the CNT at its midpoint as function of the excitation frequency. This excitation is obtained by a DC load superimposed to an AC harmonic load around one of the natural frequencies of the CNT.

In Figures 9 and 10, we plot the frequency responses of CNT near the 1st and 9th natural frequencies for initial curvature $b_{0}=100 \mathrm{~nm}$ and frequency responses of CNT near 1st and 11th natural frequencies for initial curvature $b_{0}=$
$200 \mathrm{~nm}$. We observe linear-like shape frequency-response curves in all cases due to low applied voltages $\left(V_{\mathrm{DC}}=0.01 \mathrm{~V}\right.$, $V_{\mathrm{AC}}=0.01 \mathrm{~V}$ ). The results are in agreement with the Galerkin method results reported in [29].

5.2. Modes' Veering. We study modes veering phenomenon by investigating the dynamic behavior of the CNT before and 


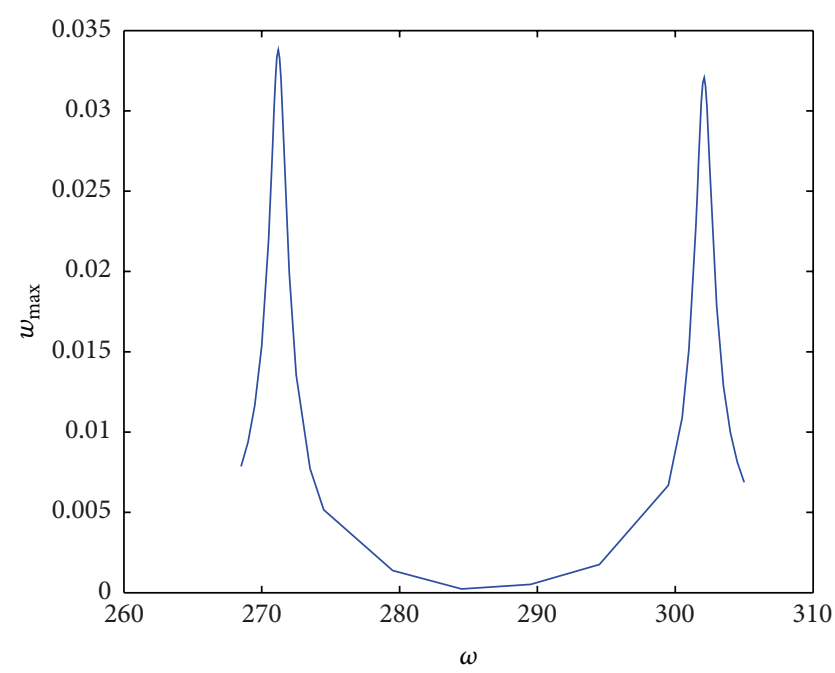

(a)

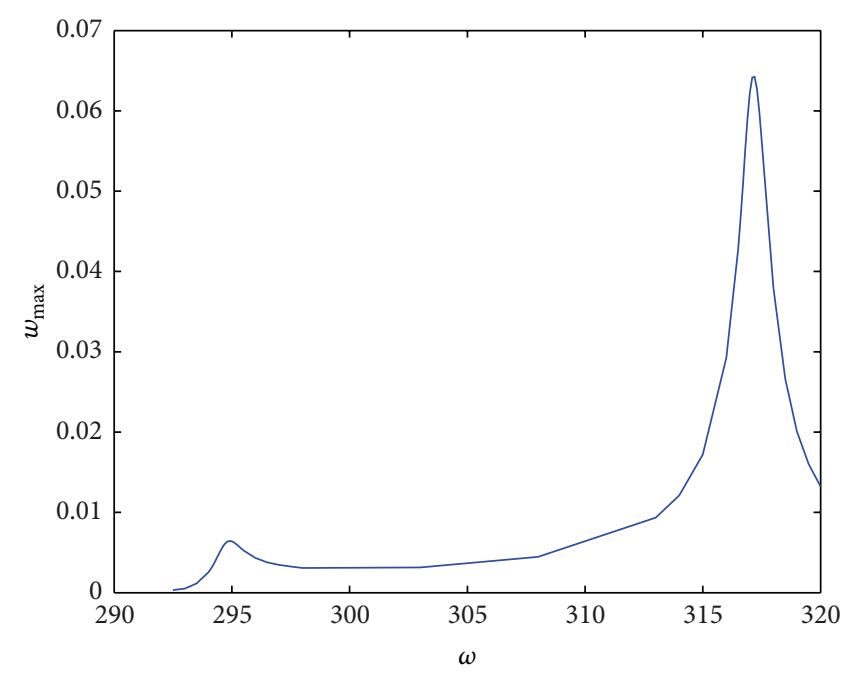

(b)

FIGURE 12: Frequency response near the third and fifth natural frequencies when third mode is excited before veering at (a) $b_{0}=25 \mathrm{~nm}$ and after veering (b) $b_{0}=40 \mathrm{~nm}$.

after veering. According to Figure 6 , the first veering between the first and third modes occurs at approximately $b_{0}=12 \mathrm{~nm}$, and the second veering occurs between the third and fifth modes at approximately $b_{0}=30 \mathrm{~nm}$.

Concerning the first veering between 1 st and 3 rd modes, we excite the CNT near its fundamental frequency and look at the response simultaneously around the first and third modes. Two peaks in the Frequency-response curve are obtained and showed in Figures 9 and 10. What we wanted to show in Figures 9 and 11 is that the linear dynamic response is significant in the neighborhood of the first natural frequency $\left(\omega_{1}\right)$ and the frequencies that are located on the veering straight line, as predicted from Figure 6. Those frequencies are $\omega_{9}$ in the case of $100 \mathrm{~nm}$ curvature and $\omega_{11}$ in the case of $200 \mathrm{~nm}$ curvature. This is actually one consequence of the mode veering where the system energy is transferred from one mode to another through the veering process. To further clarify this point, we will adopt the same previous procedure for the second veering point (between the third and the fifth natural frequencies in Figures 11 and 12) while exciting near the third natural frequency and getting the dynamic responses around the third and fifth vibration modes. In both cases after veering, the amplitude peak at the higher mode is increased compared to that before veering. The results prove that the dynamic amplitude of the CNT is a sort of exchange between two consecutive odd modes laying on the veering line. This will definitely has an effect on the system quality factor, and we may have a sort of energy exchange among the respective modes.

\section{Conclusions}

In this paper, the nonlinear dynamics of a CNT have been investigated using a novel discretization technique: FDM and DQM. In the static part, CNT responses to DC electric load were calculated solving the static equation using DQM. We have studied the static CNT behavior and how it is affected by residual stress as well as initial curvature. The natural frequencies of the CNT have been calculated for different DC voltages and initial curvatures. This study revealed the existence of the phenomenon of modes veering as both DC voltage and initial curvature are varied.

Dynamic analysis was also investigated for the CNT response to harmonic electric load near the natural frequencies. Frequency-response curves have been plotted and validated under different excitation conditions.

Veering of the modes has been analyzed by comparing the dynamic behavior of the CNT before and after veering. Veering of the modes with initial curvature as parameter is found to exchange dynamic properties between modes. In fact, the maximum deflection at resonant frequency of lower mode before veering is higher than that after veering, and maximum deflection at resonant frequency of higher mode increases considerably during veering of the modes. This proves that energy of the system is transferred from one mode to the other during veering.

\section{References}

[1] S. Iijima, "Helical microtubules of graphitic carbon," Nature, vol. 354, pp. 56-58, 1991.

[2] J. H. Bak, Y. D. Kim, S. S. Hong et al., "High-frequency micromechanical resonators from aluminium-carbon nanotube nanolaminates," Nature Materials, vol. 7, no. 6, pp. 459463, 2008.

[3] H. W. C. Postma, I. Kozinsky, A. Husain, and M. L. Roukes, "Dynamic range of nanotube- and nanowire-based electromechanical systems," Applied Physics Letters, vol. 86, no. 22, Article ID 223105, pp. 1-3, 2005.

[4] H. M. Ouakad and M. I. Younis, "Nonlinear dynamics of electrically actuated carbon nanotube resonators," AMSE Journal of 
Computational and Nonlinear Dynamics, vol. 5, no. 1, pp. 1-13, 2010.

[5] M. Dequesnes, S. V. Rotkin, and N. R. Aluru, "Parameterization of continuum theories for single wall carbon nanotube switches by molecular dynamics simulations," Journal of Computational Electronics, vol. 1, no. 3, pp. 313-316, 2002.

[6] M. Dequesnes, Z. Tang, and N. R. Aluru, "Static and dynamic analysis of carbon nanotube-based switches," AMSE Journal of Engineering Materials and Technology, vol. 126, no. 3, pp. 230237, 2004.

[7] P. Poncharal, Z. L. Wang, D. Ugarte, and W. A. de Heer, "Electrostatic deflections and electromechanical resonances of carbon nanotubes," Science, vol. 283, no. 5407, pp. 1513-1516, 1999.

[8] R. Ansari and H. Ramezannezhad, "Nonlocal Timoshenko beam model for the large-amplitude vibrations of embedded multiwalled carbon nanotubes including thermal effects," Physica E, vol. 43, no. 6, pp. 1171-1178, 2011.

[9] A. Isacsson and J. M. Kinaret, "Parametric resonances in electrostatically interacting carbon nanotube arrays," Physical Review B, vol. 79, no. 16, Article ID 165418, 2009.

[10] M. A. Hawwa and H. M. Al-Qahtani, "Nonlinear oscillations of a double-walled carbon nanotube," Computational Materials Science, vol. 48, no. 1, pp. 140-143, 2010.

[11] B. Fang, Y.-X. Zhen, C.-P. Zhang, and Y. Tang, "Nonlinear vibration analysis of double-walled carbon nanotubes based on nonlocal elasticity theory," Applied Mathematical Modelling, vol. 37, no. 3, pp. 1096-1107, 2012.

[12] A. Hajnayeb and S. E. Khadem, "Nonlinear vibration and stability analysis of a double-walled carbon nanotube under electrostatic actuation," Journal of Sound and Vibration, vol. 331, no. 10, pp. 2443-2456, 2012.

[13] P.-H. Huang, "Atomistic simulations of shearing friction and dynamic adhesion of double-walled carbon nanotubes on $\mathrm{Au}$ substrates," Composites Science and Technology, vol. 72, no. 5, pp. 599-607, 2012.

[14] A. R. Hall, S. Paulson, T. Cui, J. P. Lu, L. C. Qin, and S. Washburn, "Torsional electromechanical systems based on carbon nanotubes," Reports on Progress in Physics, vol. 75, no. 11, Article ID 116501, 2012.

[15] P. Boissy, J. Genest, J. Patenaude et al., "Carbon nanotubes (CNTs) based strain sensors for a wearable monitoring and biofeedback system for pressure ulcer prevention and rehabilitation," in Proceedings of the 33rd Annual International Conference of the IEEE Engineering in Medicine and Biology Society (EMBS '11), pp. 5824-5827, IEEE, Boston, Mass, USA, September 2011.

[16] C. Boero, J. Olivo, S. Carrara, and G. de Micheli, "A selfcontained system with CNTs-based biosensors for cell culture monitoring," IEEE Journal on Emerging and Selected Topics in Circuits and Systems, vol. 2, no. 4, pp. 658-671, 2012.

[17] H. Lorenz, J. Fritzsche, A. Das et al., "Advanced elastomer nanocomposites based on CNT-hybrid filler systems," Composites Science and Technology, vol. 69, no. 13, pp. 2135-2143, 2009.

[18] Z. Dong, U. C. Wejinya, and S. N. S. Chalamalasetty, "Development of CNT-ISFET based $\mathrm{pH}$ sensing system using atomic force microscopy," Sensors and Actuators A, vol. 173, no. 1, pp. 293-301, 2012.

[19] H. Cho, B. Jeong, M.-F. Yu, A. F. Vakakis, D. M. McFarland, and L. A. Bergman, "Nonlinear hardening and softening resonances in micromechanical cantilever-nanotube systems originated from nanoscale geometric nonlinearities," International Journal of Solids and Structures, vol. 49, no. 15-16, pp. 2059-2065, 2012.

[20] M. W. Graham, T. R. Calhoun, A. A. Green, M. C. Hersam, and G. R. Fleming, "Two-dimensional electronic spectroscopy reveals the dynamics of phonon-mediated excitation pathways in semiconducting single-walled carbon nanotubes," Nano Letters, vol. 12, no. 2, pp. 813-819, 2012.

[21] H. B. Peng, C. W. Chang, S. Aloni, T. D. Yuzvinsky, and A. Zettl, "Microwave electromechanical resonator consisting of clamped carbon nanotubes in an abacus arrangement," Physical Review B, vol. 76, no. 3, Article ID 035405, 2007.

[22] P. A. Greaney and J. C. Grossman, "Nanomechanical energy transfer and resonance effects in single-walled carbon nanotubes," Physical Review Letters, vol. 98, no. 12, Article ID 125503, 2007.

[23] F. Najar, S. Choura, S. El-Borgi, E. M. Abdel-Rahman, and A. H. Nayfeh, "Modeling and design of variable-geometry electrostatic microactuators," Journal of Micromechanics and Microengineering, vol. 15, no. 3, pp. 419-429, 2005.

[24] F. Najar, S. Choura, E. M. Abdel-Rahman, S. El-Borgi, and A. Nayfeh, "Dynamic analysis of variable-geometry electrostatic microactuators," Journal of Micromechanics and Microengineering, vol. 16, no. 11, pp. 2449-2457, 2006.

[25] F. Najar, A. H. Nayfeh, E. M. Abdel-Rahman, S. Choura, and S. El-Borgi, "Nonlinear analysis of MEMS electrostatic microactuators: primary and secondary resonances of the first mode," Journal of Vibration and Control, vol. 16, no. 9, pp. 13211349, 2010.

[26] V. Sazonova, Y. Yalsh, I. Üstünel, D. Roundy, T. A. Arlas, and P. L. McEuen, "A tunable carbon nanotube electrochemical oscillator," Nature, vol. 431, no. 7006, pp. 284-287, 2004.

[27] M.-F. Yu, "Fundamental mechanical properties of carbon nanotubes: current understanding and the related experimental studies," ASME Journal of Engineering Materials and Technology, vol. 126, no. 3, pp. 271-278, 2004.

[28] H. M. Ouakad and M. I. Younis, "Dynamic response of slacked single-walled carbon nanotube resonators," Nonlinear Dynamics, vol. 67, no. 2, pp. 1419-1436, 2012.

[29] H. M. Ouakad and M. I. Younis, "Natural frequencies and mode shapes of initially curved carbon nanotube resonators under electric excitation," Journal of Sound and Vibration, vol. 330, no. 13, pp. 3182-3195, 2011.

[30] A. Bhushan, M. M. Inamdar, and D. N. Pawaskar, "Investigation of the internal stress effects on static and dynamic characteristics of an electrostatically actuated beam for MEMS and NEMS application," Microsystem Technologies, vol. 17, no. 12, pp. 17791789, 2011.

[31] H. Ouakad and M. I. Younis, "Internal resonance in electrically actuated MEMS arches," in Proceeding of the 7th European Nonlinear Dynamics Conference (ENOC '11), Rome, Italy, 2011. 


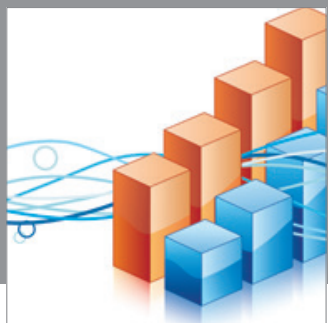

Advances in

Operations Research

mansans

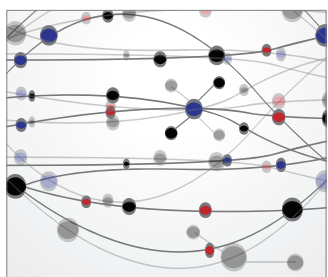

The Scientific World Journal
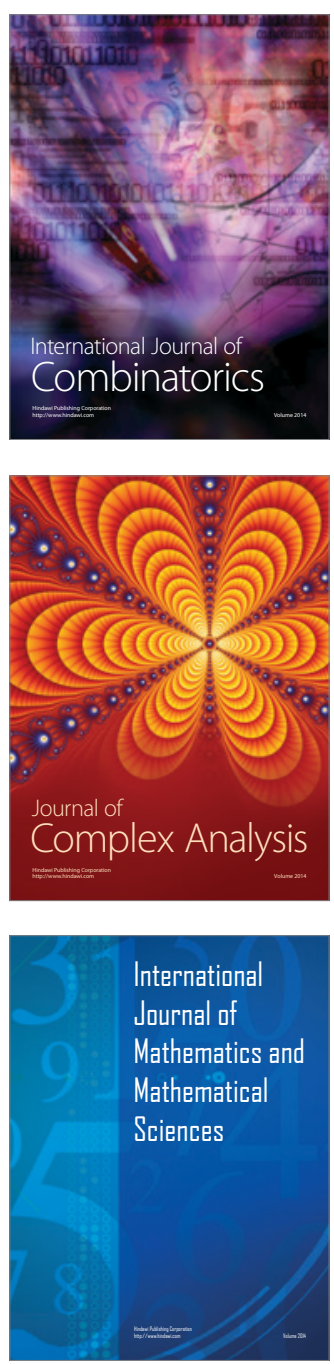
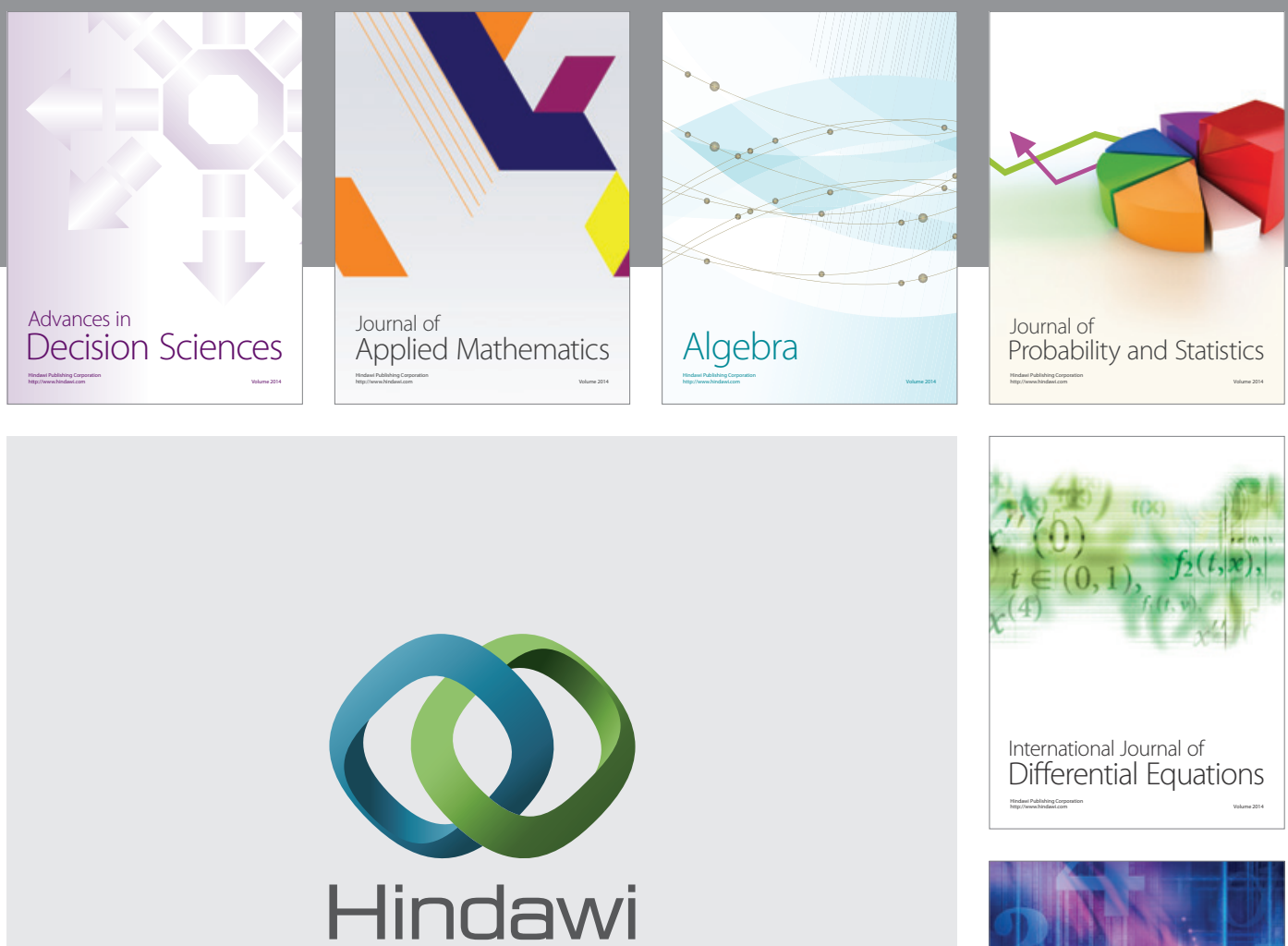

Submit your manuscripts at http://www.hindawi.com
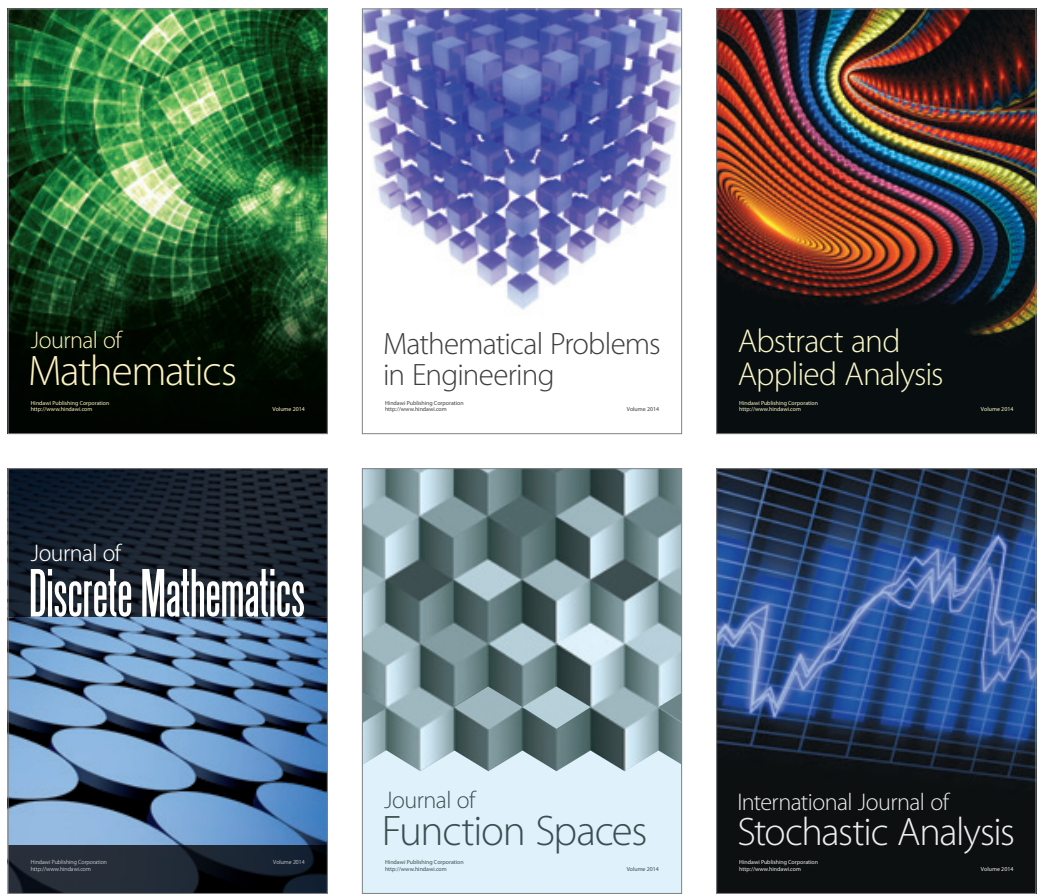

Journal of

Function Spaces

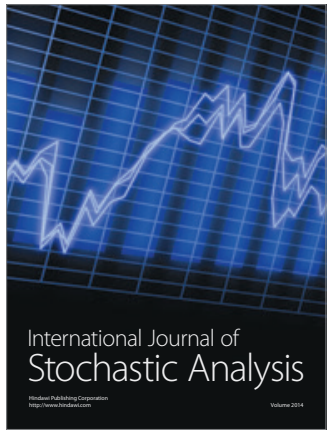

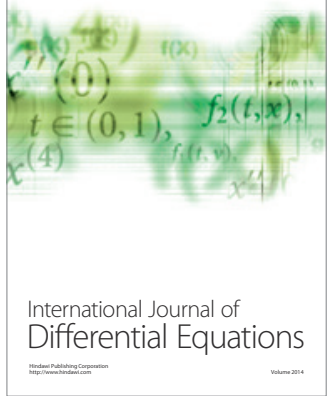
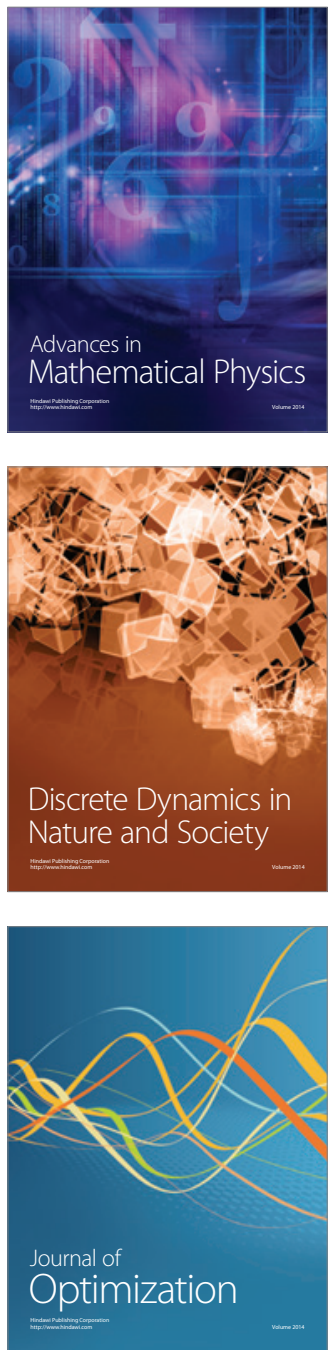